Science, Technology and Development 34 (2): 82-85, 2015

ISSN 0254-6418 / DOI: 10.3923/std.2015.82.85

(C) 2015 Pakistan Council for Science and Technology

\title{
Animal Models for Pre-Clinical Antiepileptic Drug Research
}

\author{
Lokesh Shetty Jagannatha \\ Department of Pharmacy and Pharmacology, \\ Riyadh Colleges of Dentistry and Pharmacy, \\ P.O. Box 84891, Riyadh, 11681, Kingdom of Saudi Arabia
}

\begin{abstract}
Animal models are essential in preclinical anti epileptic drug research. These models serve different purposes in epilepsy research and in drug discovery and development of anti epileptic drugs to evaluate efficacy, to determine mechanism of action, used to determine preclinical efficacy of new drugs during chronic administration, to study the mechanism of drug resistance and also used to study whether eplileptogenesis alters the adverse effect potential of a given drug. A large number of in vivo animals models are used, some important which can be adapted and acclimatized to institutional laboratory settings discussed.
\end{abstract}

Key words: Epileptogenesis, In vivo animal models, antiepileptic

\section{INTRODUCTION}

The animal models are essential in the pre-clinical research of new drugs to develop new treatment approaches for epileptic seizures. In epilepsy research animal models serve different purposes. They are used in development of new epileptic drugs, used to evaluate specific efficacies of drugs on different types of seizures and used to determine preclinical efficacy of new drugs during chronic administration. Animal models are also employed to study the pharmacodynamics, mechanism of drug resistance and also used to study whether eplileptogenesis alters the adverse effect potential of a given drug. Most epilepsy models used in epilepsy research are models of epilepsy seizures than models of epilepsy. Since epilepsy is characterized by spontaneous recurrent seizures, models such as maximal electroshock seizure, in which an acute seizure is electrically induced in normal non epileptic animals, cannot represent a model of epilepsy. On other hand, there are true models of epilepsy, for instance animal mutants or transgenic animals with spontaneously recurrent seizures, are very close to human epilepsy models (Lothman, 1996).

An Ideal animal model of epilepsy should fulfill to develop spontaneously occurring recurrent seizure and seizure type produced should be similar to those occurring in human epilepsies with high seizure frequency to allow acute and chronic drug efficacy study. But no animal models meet all these criteria, however for practical reasons, the most commonly used animal models in preclinical anti epileptic drug research are not models of chronic epilepsy but models of single epileptic seizures, in which seizures are induced in small laboratory by using rats and mice with simple chemical or electrical stimuli (Loscher, 1984, 1992, 1999).
Animal models used in anti-epileptic drug research: In order to study the antiepileptic effect of drugs and discovering their mechanism of action, various in vitro and in vivo models of epilepsy have been devised. Some of them which are used most often are described below.

Maximal electroshock (MES) induced convulsions: Merritt and Putnam developed the MES test and discovered the anticonvulsive effect of diphenyl hydantoin using this test (Putnam and Merritt, 1937). Phenytoins subsequent success in the clinical management of generalized tonic clonic seizures provided the validation necessary to consider the MES test as a reasonable model of human generalized tonic clonic seizure (White, 2003). The MES produce the spread of seizure similar to grandmal epilepsy (Kale and Kale, 1994). In MES method brief high intensity shock is applied to the head through corneal or ear electrodes with a stimulator that either delivers constant current on constant voltage as a frequency of $50-60 \mathrm{sec}^{-1}$. The electrodes are moistened with saline solution before application for better conductance. All animals are stimulated with the constant current stimulator typical stimulation parameters include $50 \mathrm{~mA}$ in mice and $150 \mathrm{~mA}$ in rats, 50-60 $\mathrm{sec}^{-1}$ current delivered via corneal/ear electrodes for $0.2 \mathrm{sec}$ (Gupta, 2004). The MES convulsions are divided into five phases such as Phase of tonic limb flexion, Phase of tonic limb extension, Phase of clonic convulsions, Stupor and Recovery or death. A substance is known to possess anticonvulsant property it reduces or abolishes the extensor phase of MES convulsion (Kulkarni, 1999). Suppression of tonic hind limb extension is taken as a measure of efficacy in this test. Anti convulsant potency is determined by calculation of $\mathrm{ED}_{50}$ for suppression of tonic hind limb extension (Swinyard, 1969). Drugs 
effective against generalized tonic clonic seizure such as Phenytoin, Carbamazepine, Phenobarbitone and Primidone are effective while Ethosuximide is in effective in this test (Gupta, 2004). Disadvantage of the present model is that, they do not give any clue regarding the mechanism of action of the compound.

Pentylene tetrazole (PTZ) induced convulsions: Pentylene tetrazole is a tetrazole derivative with consistent convulsive effect in large number of animals like mice, rats, cats, primate's etc. It is believed to act by antagonizing the inhibitory GABAergic neurotransmission (Olsen, 1981). In 1944, Everett and Richards's demonstrated that PTZ-induced seizures could be blocked by Trimethadione and Phenobarbital but not by Phenytoin. Lennox subsequently demonstrated that Trimethadione was effective in decreasing or preventing petitmal attacks in 50 patients but was ineffective or worsened grandmal attacks in patients. Trimethadione success in the clinic and its ability to block threshold seizures induced by PTZ provided the necessary correlation to establish the PTZ test as model of petitmal or generalized absence seizure (White, 2003; Lennox, 1945). Pharmacologically PTZ is an analeptic agent; possible common mechanism of action is ability to alter movement of chloride ions across neuronal membrane (Kaminski et al., 2007). It is reported that mice lacking $\delta$ subunit exhibit spontaneous seizure and greater sensitivity PTZ, demonstrating a role of the $\delta$ subunit containing $\mathrm{GABA}_{\mathrm{A}}$ receptor in regulating seizure susceptibility (Meldrum and Rogawski, 2007). It is believed to be PTZ act by antagonizing the inhibitory GABAergic neurotransmission but binding site is unknown (Huang et al., 2001). Different routes of administration such as i.p, i.v, s.c have been used by various investigations in this method (Vogel and Vogel, 2002). Stages of PTZ induced convulsion involve one or more isolated jerks followed immediately by a generalized colic seizure with loss of righting reflex, followed by maximum tonic clonic seizures after a certain time lag. Efficacy of test drug as an anticonvulsant is measured by determining its $\mathrm{ED}_{50}$ for suppression of clonic seizures. Drugs effective in petitmal epilepsy like valproic acid, ethosuximide are effective in PTZ induced convulsion while phenytoin, carbamazepines are not effective (Gupta, 2004).

Lithium-pilocarpine induced status epilepticus: Although, lithium does not have general proconvulsant action in rats, its pretreatment provokes limbic seizures, following administration of subconvulsant doses of Pilocarpine and other cholinergic agonists. The time of onset of severity of seizures is reproducible and generalized. The convulsions produced always continue unabated for several hours until death ensures. It is reported that the initiation of seizure is due to activation of muscarinic cholinergic receptors but the mechanism accounting for the sustained maintenance of status Epilepticus are not known, although there is the evidence for the involvement of the excitatory amino acids. The combined treatment with lithium and pilocarpine results in an accumulation of inositol monophosphate and reduction in cortical inositol those are about ten times greater than the effects obtained with either drug alone (Kasture and Kasture, 1999). Status epilepticus can be induced in rats by giving pilocarpine (30-40 $\mathrm{mg} \mathrm{kg}^{-1}$, i.p) $24 \mathrm{~h}$ after pre treating with lithium (3 meq $\mathrm{kg}^{-1}$, i.p) (Jope et al., 1986; Morrisett et al., 1987; Walton and Trieman, 1988).

Lithium-methomyl induced seizure in rats: Methomyl, a carbamate anticholinesterase, in a dose of $5.2 \mathrm{mg} \mathrm{kg}^{-1}$ s.c, can induce long lasting status epilepticus in lithium pretreated rats. Diazepam is effective in this model (Kaminski et al., 2007).

Isoniazid induced seizures: At higher dose of Isoniazid acts as convulsant in animal models. The mechanism of Isoniazid triggered convulsion involves interaction with pyridoxine metabolism. The mechanism responsible for epileptogenesis is interfering with the function and supply of pyridoxine. Isoniazid binds directly with pyridoxine to form Isonicotinyl hydrazine. The second depicted mechanism is Isoniazid is dehydrised to its hydrazones which block pyridoxine phosphokinase, thus preventing conversion of pyridoxine to its active from, pyridoxal 5' phosphate. Isoniazid also hydrazides inactivate pyridoxal 5 phosphate, which is essential for the formation of gamma amino-butyric acid from glutamic acid. Deficient GABA and the accumulation of glutamic acid lead to CNS excitation and seizures in animal models (Vaishal and Agarwal, 2004). Intraperitoneal administration of $300 \mathrm{mg} \mathrm{kg}^{-1}$ of Isoniazid induces seizures in mice (Madhu et al., 2009).

Systemic penicillin test: The possible mechanism of eplileptogenesis of penicillin is via blocking of the GABA's effect when the beta lactam ring binds to GABA receptor. Cats are used for the experiment. Animals are injected with 300000 units $\mathrm{kg}^{-1}$ of penicillin G by intramuscular route. Seizure activity begins one hour after injection. The seizures are characterized by recurrent episodes of arrested activity, staring, myoclonus, facial oral twitching and occasional progression to generalized tonic clonic seizures (Taylor-Courval and Gloor, 1984). This model is useful for screening of drugs useful in petitmal epilepsy. Ethosuximide and valproate are effective in this model (Pellegrini et al., 1978; Guberman et al., 1975). Rats also can be used for the penicillin model of epilepsy (Fariello et al., 1976). 
Selection, handling and dosing for animal species for anti-epileptic screening: The preferred rodent species is the rat although other rodent species may be used for study such as mice. Normally female rats or mice are preferred (OECD., 2000). This is because literature surveys shows that usually there is little difference in sensitivity between sexes. But in those cases where differences are observed, females are generally slightly more sensitive than male species (Lipnick et al., 1995). Healthy young adult animals of laboratory strains should be used. Females should be nulliparous and non-pregnant. At the beginning of dosing, each animal should be between 8 and 12 weeks old and its weight should fall in an interval within $\pm 20 \%$ of the mean initial weight of any previously dosed animals. The temperature of experimental animal room should be maintained at $22^{\circ} \mathrm{C}\left( \pm 3^{\circ} \mathrm{C}\right)$. The relative humidity should be at least $30 \%$ and not to exceed $70 \%$. Lighting should be provided artificially, the sequence being $12 \mathrm{~h}$ light and $12 \mathrm{~h}$ dark. The animals are housed individually. For feeding, conventional rodent laboratory diets may be used. Animals should be provided with an unlimited supply of drinking water. The animals are randomly selected, marked to permit individual identification and kept in their cages for at least 5 days prior to dosing to allow for acclimatization to the laboratory conditions. As with other sequential test designs, care must be taken to ensure that animals are available in the appropriate size and age range for the entire study (Shetty et al., 2009).

The test substance is administered in a single dose by gavage using a stomach tube or a suitable intubation cannula. In the unusual circumstance that a single dose is not possible, the dose may be given in smaller fractions over a period not exceeding $24 \mathrm{~h}$. Selection of test dose for preliminary dosing should be done in such way that, middle dose will be approximately one tenth of the maximum dose during acute toxicity studies and a high dose will be twice that of one tenth doses and a low dose will be $50 \%$ of one tenth doses (OECD., 2000).

\section{CONCLUSION}

A large number of in vivo animal models for screening of antiepileptic drugs are available. There are no models that satisfy as an ideal model in epilepsy research. In epilepsy research, animal models serve a variety of purposes. They are used in the search for new antiepileptic drugs. Once the anticonvulsant activity of a novel compound has been detected, animal models are used to evaluate the possible specific efficacies of the compound against different types of seizures or epilepsy. The animal models can be used to characterize the preclinical efficacy of novel compounds during chronic administration. The animal models which can be adapted and acclimatized for institutional laboratory settings are of chemical and electrical stimuli models.

\section{REFERENCES}

Fariello, R.G., A. Portera and D. Scheffner, 1976. Parenteral penicillin in rats: An experimental model of multifocal epilepsy. Epilepsia, 17: 217-222.

Guberman, A., P. Gloor and A.L. Sherwin, 1975. Response of generalized penicillin epilepsy in the cat to ethosuximide and diphenylhydantoin. Neurology, 25: 758-758.

Gupta, S.K., 2004. Drug Screening Methods. Jaypee Brothers Medical Publishers Ltd., New Delhi, India, ISBN-13: 9788180613975.

Huang, R.Q., C.L. Bell-Horner, M.I. Dibas, D.F. Covey, J.A. Drewe and G.H. Dillon, 2001. Pentylenetetrazole-induced inhibition of recombinant $\gamma$-aminobutyric acid type A (GABAA) receptors: Mechanism and site of action. J. Pharmacol. Exp. Therap., 298: 986-995.

Jope, R.S., R.A. Morrisett and O.C. Snead, 1986. Characterization of lithium potentiation of pilocarpine-induced status epilepticus in rats. Exp. Neurol., 91: 471-480.

Kale, S.R. and K.R. Kale, 1994. Practical Pharmacology. Nirali Prakashan, India.

Kaminski, R.M., P. Blaszczak, A. Dekundy, J. Parada-Turska, L. Calderazzo, E.A. Cavalheiro and W.A. Turski, 2007. Lithium-methomyl induced seizures in rats: A new model of status epilepticus?. Toxicol. Applied Pharmacol., 21 219: 122-127.

Kasture, S.B. and V.S. Kasture, 1999. Effect of some serotonergic agents on lithium-pilocarpine model of status epilepticus in rats. Indian J. Pharmacol., 31: 370-372.

Kulkarni, S.K., 1999. Handbook of Experimental Pharmacology. 3rd Edn., Vallabh Prakashan, New Delhi, India, ISBN: 8185731128, pp: 133-134.

Lennox, W.G., 1945. The petit mal epilepsies: Their treatment with tridione. J. Am. Med. Assoc., 129: 1069-1074.

Lipnick, R.L., J.A. Cotruvo, R.N. Hill, R.D. Bruce and K.A. Stitzel et al., 1995. Comparison of the up-and-down, conventional $\mathrm{LD}_{50}$ and fixed-dose acute toxicity procedures. Food Chem. Toxicol., 33: 223-231.

Loscher, W., 1984. Genetic animal models of epilepsy as a unique resource for the evaluation of anticonvulsant drugs: A review. Meth. Findings Exp. Clin. Pharmacol., 6: 531-547.

Loscher, W., 1992. Genetic Animal Models of Epilepsy. In: Genetically Defined Animal Models of Neurobehavioral Dysfunctions, Driscoll, P. (Eds.). Birkhauser, Boston, Massachusetts, pp: 111-135. 
Loscher, W., 1999. Animal Models of Epilepsy and Epileptic Seizures. In: Handbook of Experimental Pharmacology, Eadie, M.J. (Ed.). Springer, Berlin, pp: 19-62.

Lothman, E.W., 1996. Neurobiology as a basis for rational polypharmacy. Section overview for rational polypharmacy conference. Epilepsy Res., 11: 3-7.

Madhu, A., P. Keerthi and S. Jaideep, 2009. Antiepileptic activity of aqueous root extract of Hemidesmus indicus in rats. Arch. Pharm. Sci. Res., 1: 43-47.

Meldrum, B.S. and M.A. Rogawski, 2007. Molecular targets for antiepileptic drug development. Neurotherapeutics, 4: 18-61.

Morrisett, R.A., R.S. Jope and O.C. Snead, 1987. Effects of drugs on the initiation and maintenance of status epilepticus induced by administration of pilocarpine to lithium-pretreated rats. Exp. Neurol., 97: 193-200.

OECD., 2000. Guidance document on acute oral toxicity. Environmental Health and Safety Monograph Series on Testing and Assessment No 24.

Olsen, R.W., 1981. The GABA postsynaptic membrane receptor inophore complex. Mol. Cell Biochem., 39: 261-279.

Pellegrini, A., P. Gloor and A.L. Sherwin, 1978. Effect of valproate sodium on generalized penicillin epilepsy in the cat. Epilepsia, 19: 351-360.
Putnam, T.J. and H.H. Merritt, 1937. Experimental determination of the anticonvulsant properties of some phenyl derivatives. Science, 85: 525-526.

Shetty, L.J., H. Harikiran and J. Fernandes, 2009. Pharmacological evaluation of ethanolic extract of flowers of Tagetes erecta on epilepsy. J. Pharm. Res., 2: 1035-1038.

Swinyard, E.A., 1969. Laboratory evaluation of antiepileptic drugs: Review of laboratory methods. Epilepsia, 10: 107-119.

Taylor-Courval, D. and P. Gloor, 1984. Behavioral alterations associated with generalized spike and wave discharges in the EEG of the cat. Exp. Neurol., 83: 167-186.

Vaishal, A.D. and S.B. Agarwal, 2004. Isoniazid toxicity. Indian Acad. Clin. Med., 5: 83-85.

Vogel, G.H. and W.H. Vogel, 2002. Drug Discovery and Evaluation of Pharmacological Assay. Springer Varlag Berlin Heidelburg, New York.

Walton, N.Y. and D.M. Treiman, 1988. Response of status epilepticus induced by lithium and pilocarpine to treatment with diazepam. Exp. Neurol., 101: 267-275.

White, H.S., 2003. Preclinical development of antiepileptic drugs: Past, present and future directions. Epilepsia, 44: 2-8. 